\title{
Study of plasma adiponectin and insulin resistance in subjects with non-alcoholic fatty liver disease.
}

\section{Hady Gad*}

Department of Internal Medicine, Middle East Medical Center, Kingdom of Bahrain

\begin{abstract}
Background: The relation between insulin resistance and the level of plasma adiponectin hormone in patients with non-alcoholic fatty liver disease (NAFLD) was highly suggested due to the high prevalence of NAFLD in obese and diabetic patients.

Objectives: This study was conducted to explore the link between the three factors, NAFLD, insulin resistance and the level of plasma adiponectin.

Methods: The study was run on patients with the diagnosis of NAFLD, depending on the ultrasound findings, with exclusion of diabetic, obese, and alcoholic patients. The level of insulin resistance was evaluated using the homeostasis model assessment (HOMA IR) index, and the level of plasma adiponectin was measured as well.

Results: There was a significant degree of insulin resistance in patients with NAFLD, with a significantly lower levels of adiponetin hormone compared to the control group.

Conclusion: This study reported lower plasma adiponectin levels in NAFLD patients that were inversely correlated with insulin resistance, which was markedly documented in the patients of NAFLD.
\end{abstract}

Keywords: Non-alcoholic fatty liver disease (NAFLD), Insulin resistance, Adiponectin hormone

\section{Introduction}

Non-alcoholic fatty liver disease (NAFLD) is a clinicopathological syndrome characterized by hepatic steatosis with or without active inflammation in patients with a negligible alcohol intake, NAFLD can progress to fibrosis and even cirrhosis and eventually can cause hepatocellular carcinoma. NAFLD is recognized as one of the leading causes of chronic liver disorders with a worldwide distribution.

Insulin resistance; a state in which a given concentration of insulin produces a less than expected biological effect. And it has been defined as the requirement of 200 or more units of insulin per day to attain glycemic control and prevent ketosis.

The homeostasis model assessment (HOMA) has been suggested as a method to assess insulin resistance using fasting blood glucose and fasting insulin concentrations.

HOMA index = fasting glucose $($ in $\mathrm{mg} / \mathrm{dl}) / 18 \mathrm{x}$ fasting insulin (in micro-unit/ml) / 22.5.

It is concluded that HOMA provides a useful model to assess insulin resistance in epidemiological studies in which only fasting samples are available [1].

According to the homeostasis model assessment (HOMA), patient is considered to have insulin resistance if HOMA index is more than 2.14 , it is thought to be the pathognomonic condition responsible for NAFLD.

Adiponectin is a hormone that is secreted by the adipocytes and it was reported that it has several anti-atherogenic, antidiabetic and anti-inflammatory properties. Experimental and clinical studies have stated that adiponectin concentrations have a positive correlation with insulin sensitivity.

This study aimed to investigate the relation between NAFLD and both of insulin resistance and adiponectin hormone and discover their role in the pathogenesis of the disease.

\section{Methodology}

The study included forty patients (twenty four females and sixteen males) with NAFLD as well as ten age and sex matched healthy volunteers serving as controls (five females and five males). Patients were selected from those attending the outpatient clinics of the internal medicine department of Benha University Hospitals.

Exclusion criteria: alcohol consumption (at any rate), patients with obesity $(\mathrm{BMI}=$ or $>30)$, evidence of viral or toxic hepatitis, patients with diabetes mellitus (fasting blood sugar $>126 \mathrm{mg}$ / dl), patients with hypertention (blood pressure $>140 / 90$ ), patients with nutritional disorders (e.g. obesity, starvation, gastrointestinal surgery for obesity, total parentral nutrition), patients who are consuming some drugs (e.g. glucocorticoid, synthetic estrogen, amiodarone, calcium channel blockers), metabolic or genetic causes of fatty liver (e.g. pregnancy, and lipodystrophy), and patients who are over 70 years.

All patients were subjected to a thorough history taking and a complete clinical examination, with special stress on the following:

Abdominal ultrasound to detect patients with NAFLD, fasting blood glucose, liver function tests [alanine aminotransferase 
(ALT) and aspartate aminotransferase (AST)], fasting serum insulin, fasting plasma adiponectin hormone, estimation of insulin resistance according to (HOMA test).

\section{Results}

\section{Laboratory findings}

- Patients with NAFLD had a significantly lower levels of plasma adiponectin compared to the control group (in the NAFLD patients the mean was 4.79 , while in the control group it was 11.8 microgram $/ \mathrm{ml}$ ) using the students $(\mathrm{t})$ test, and significance was adopted at $\mathrm{p}<0.05$, (Table 1 ).

- Patients with NAFLD had higher levels of fasting blood glucose, fasting serum insulin and insulin resistance (HOMA test) compared to the control group, using the students ( $\mathrm{t}$ ) test, and significance was adopted at $\mathrm{p}<0.05$, (Table 2).

- This study demonstrated that plasma adiponectin levels were higher in females than in males (in females the mean was 3.14, while in males it was 2.26 microgram/ $\mathrm{ml}$ ) using the students ( $\mathrm{t})$ test, and significance was adopted at $\mathrm{p}<0.05$, (Table 3 ).

- Plasma Adiponectin level was negatively correlated with: fasting blood glucose, fasting serum insulin level, and insulin resistance (HOMA test), with Spearman's rank correlation (r) $-0.671,-0.426$, and -0.66 respectively, (Table 4).

\section{Discussion}

Non-alcoholic fatty liver disease (NAFLD) is emerging as one of the most common liver disorders claiming the urgent attention

Table 1. Range and mean $\pm S D$ (standard deviation) of plasma adiponectin in the studied groups and their statistical difference.

\begin{tabular}{|l|c|c|c|c|}
\hline & $\begin{array}{c}\text { Patients group } \\
\text { (NAFLD) } \\
\text { "n=40" }\end{array}$ & $\begin{array}{c}\text { Control group } \\
\text { "n=10" }\end{array}$ & $\mathbf{t}$ & $\mathbf{p}$ \\
\hline $\begin{array}{l}\text { Plasma Adiponectin } \\
\text { (microgram/ml) }\end{array}$ & & & & \\
$\begin{array}{l}\text { Range } \\
\text { Mean }\end{array}$ & $3-13$ & $10.5-13$ & & \\
\pm IS.D. & 4.79 & 11.8 & & \\
\hline
\end{tabular}

Table 2. Range and mean $\pm S D$ (standard deviation) of fasting blood glucose, fasting serum insulin and insulin resistance in the studied groups and their statistical difference.

\begin{tabular}{|c|c|c|c|c|}
\hline & $\begin{array}{l}\text { Patients group } \\
\text { (NAFLD) } \\
\text { "n=40" }\end{array}$ & $\begin{array}{c}\text { Control group } \\
\qquad n=10 "\end{array}$ & $\mathbf{t}$ & $\mathbf{p}$ \\
\hline $\begin{array}{l}\text { Fasting blood } \\
\text { glucose (mg/dl) } \\
\text { Range } \\
\text { Mean } \\
\text { 土S.D. }\end{array}$ & $\begin{array}{c}70-120 \\
83.70 \\
10.28\end{array}$ & $\begin{array}{c}65-100 \\
78.4 \\
11.56\end{array}$ & 1.65 & $>0.05$ (N.S.) \\
\hline $\begin{array}{l}\text { Fasting } \\
\text { serum insulin } \\
\text { (microunit/ml) } \\
\text { Range } \\
\text { Mean } \\
\text { 土S.D. }\end{array}$ & $\begin{array}{c}3.599-34.557 \\
11.21 \\
6.81\end{array}$ & $\begin{array}{c}4.67-15.73 \\
7.79 \\
3.21\end{array}$ & 2.98 & $<0.05(\mathrm{~S})$ \\
\hline $\begin{array}{l}\text { Insulin } \\
\text { resistance } \\
\text { (HOMA test) } \\
\text { Range } \\
\text { Mean } \\
\pm \text { S.D. }\end{array}$ & $\begin{array}{c}0.37-10.58 \\
2.73 \\
2.06\end{array}$ & $\begin{array}{c}0.816-1.23 \\
1.01 \\
0.79\end{array}$ & 5.11 & $<0.05$ (S) \\
\hline
\end{tabular}

of both medical professionals and the public sphere, as it is revealed that simple triglyceride accumulation in hepatocytes (steatosis) frequently becoming complicated by inflammation (non-alcoholic steatohepatitis or NASH) that may progress into more advanced stages of the disease including cirrhosis or, eventually, hepatocellular carcinoma. NAFLD is intimately associated with progressive manifestation of insulin resistance in the liver, and it was approved that insulin resistance in liver and adipose tissue precedes the impairment of insulin sensitivity in skeletal muscle [2].

Adiponectin is a fat derived hormone, that is produced by the adipocytes [3]. It is an anti-diabetic and anti-atherogenic hormone that correlates with insulin sensitivity. Adiponectin directly counteracts the effect of tumor necrosis factor alpha (TNF- $\alpha$ ) on insulin signaling and lipid metabolism [4].

The discovery of adiponectin has added an additional potential mechanism to explain the pathogenesis of liver steatosis [5]. Adiponectin is a relatively abundant serum hormone which was reported to influence both lipid and glucose metabolism in the liver and in muscle tissue. In the liver, it increases the sensitivity to insulin so it inhibits gluconeogenesis and regulates hepatic non-estrified fatty acid metabolism, via suppression of lipogenesis and activation of non-estrified fatty acid oxidation. It is proposed that adiponectin enhances expression of the peroxisome-proliferator activated receptor alpha (PPAR- $\alpha$ ) gene, leading to increased fat oxidation [6]. In healthy adults, a relationship was observed between adiponectin deficiency and increased liver fat [7]. It is found that in mild obesity, hypoadiponectinemia predicted the presence of hepatic steatosis at abdominal ultrasound [8]. Adiponectin has antiinflammatory properties in the liver, and its deficiency might account for high aminotransferase and liver disease progression [6], it may be able to preserve liver function by preventing lipid accumulation in hepatocytes [9]. Moreover a direct antifibrotic effect of adiponectin has been suggested on the basis of adiponectin receptor gene expression in hepatic stellate cells and the inhibition of stellate cells proliferation and migration after adiponectin treatment [10], it also inhibits tumor necrosis

Table 3. Mean $\pm S D$ (standard deviation) of plasma adiponectin in females and males in both groups (NAFLD group and control group) and their statistical difference.

\begin{tabular}{|c|c|c|c|c|}
\hline & $\begin{array}{c}\text { Females } \\
\text { "n=29" }\end{array}$ & $\begin{array}{c}\text { Males } \\
\text { “n=21" }\end{array}$ & $\mathbf{t}$ & $\mathbf{p}$ \\
\hline $\begin{array}{c}\text { Plasma adiponectin } \\
\text { (microgram/ml) }\end{array}$ & & & & \\
Mean & 3.14 & 2.26 & & \\
IS.D. & 1.81 & 1.07 & 1.85 & $<0.05(\mathrm{~S})$ \\
\hline
\end{tabular}

Table 4. Correlation between plasma adiponectin levels (microgram/ $\mathrm{ml}$ ) and fasting blood glucose (mg/dl), fasting insulin (microunit/ml), and insulin resistance.

\begin{tabular}{|c|c|c|}
\hline \multirow{2}{*}{} & \multicolumn{2}{|c|}{ Adiponectin } \\
\cline { 2 - 3 } & $\mathbf{r}$ & $\mathbf{p}$ \\
\hline FBG $(\mathrm{mg} / \mathrm{dl})$ & -0.671 & $<0.05(\mathrm{~S})$ \\
\hline Fasting serum insulin (microunit/ml) & -0.426 & $<0.05(\mathrm{~S})$ \\
\hline insulin resistance (HOMA test) & -0.66 & $<0.05(\mathrm{~S})$ \\
\hline
\end{tabular}


factor alpha (TNF- $\alpha$ ) expression and prevents the expression of cytokines in hepatic stellate cells and it may protect against steatohepatitis through its anti-inflammatory action, as it is well known that inflammation is a key mechanism in the progression of fatty liver to steatohepatitis and cirrhosis [5].

In this study the plasma adiponectin levels were significantly lower in the NAFLD patients group, this result agrees with other studies; Bugianesi et al. [6], Pagano et al. [5], Sargin et al. [9] and Yoon et al. [11]. And this reduction was associated with insulin resistance. The strong association between insulin resistance and NAFLD has been extensively demonstrated [5].

In this study the levels of plasma adiponectin were significantly higher in females than in males, this observation was supported by Yamamoto et al. [12] who stated that adiponectin concentrations seem to be gender-dependent, being higher in women than in men. This fact was also documented in many other studies; Bugianesi et al. [6], Sargin et al. [9] and Yoon et al. [11] who stated higher adiponectin expression in women, as compared to men.

The study showed that patients with NAFLD had significantly increased values of fasting serum insulin and insulin resistance (HOMA test) compared to the control group, while there was a non significant change in the fasting glucose levels in both groups. This result is advocated for the statement that, insulin resistance is the pathognomonic condition responsible for NAFLD [11]. And this notion was supported by Bloomgarden [13] who stated that individuals with NAFLD have the same degree of insulin resistance as those with type 2 diabetes mellitus.

In agreement with this study Pagano et al. [5] demonstrated the strong association between insulin resistance and NAFLD, it was shown that fasting serum insulin and insulin resistance (HOMA test) levels were significantly higher in NAFLD patients than in control subjects.

Bugianesi et al. [6] enforced this result as it was found that patients with NAFLD had higher serum insulin levels and insulin resistance (HOMA test) than the control group despite of the similar glucose tolerance in the two groups.

In a population-based study of $>2,000$ individuals having magnetic resonance spectroscopy, more than one-third of the population had evidence of elevated hepatic triglycerides, with $30 \%$ of these individuals having insulin resistance syndrome, suggesting insulin resistance to be the primary factor in inducing fatty liver [14].

This result was also supported by the study of Sargin et al. [9], which revealed that levels of fasting insulin and insulin resistance were statistically and significantly higher in NAFLD patients than in the control group.

Insulin resistance is the most reproducible factor in the development of NAFLD [15] and it is a virtually universal feature of NASH that occurs independently of obesity, as insulin resistance leads to chronic hyperinsulinemia due to an impaired response of adipose, muscle, and liver tissue to the glucoregulatory effects of insulin. Insulin resistance at the level of the adipocyte further exacerbates excess fatty acids delivery through ongoing lipolysis in the fed state, where under normal circumstances lipolysis would be inhibited. In addition, hyperinsulinemia stimulates de novo lipogenesis and can impair fatty acids oxidation and very low density lipoprotein (VLDL) secretion. This excessive intrahepatic lipid accumulation impairs insulin signaling (hepatic insulin resistance). In the setting of hepatic insulin resistance, gluconeogenesis is stimulated despite high insulin levels, thus contributing to the already distorted glucose metabolism [4].

This study found that there was a significant negative correlation between plasma adiponectin levels and fasting blood glucose, fasting serum insulin levels and insulin resistanc (HOMA test). This is agreed with Yamamoto et al. [12], Bugianesi et al. [6], Pagano et al. [5] and Yoon et al. [11].

\section{Conclusion}

In conclusion, this study reported lower adiponectin levels in NAFLD patients that were inversely correlated with insulin resistance, which was markedly documented in the patients of NAFLD. These data support that insulin resistance is a primary pathognomonic factor in NAFLD and also support a role for adiponectin hormone in the protection against liver injury, in the context of the hypothesis that an imbalance between the pro-inflammatory and the anti-inflammatory cytokines may have a pathogenic role in the development of the liver damage in NAFLD. Moreover the relation between the adipose tissue and the liver may act as a major player in the link between the metabolic syndrome and the NAFLD.

Adiponectin may be applicable in human diseases as a novel agent for diagnosis and treating insulin resistance and NAFLD, as increased levels of the hormone have been correlated to improving histopathology of the liver, suggesting that it may play an important role in the progression from NAFLD to $\mathrm{NASH}$, in NASH patients with a positive histological response to treatment, increased levels of adiponectin could be seen in as early as 1 to 3 months post-treatment. Levels continued to rise throughout the course of therapy. On the contrary, as demonstrated by another study, patients receiving a placebo experienced minimal changes, while non-responders showed decreased adiponectin levels [16,17].

\section{References}

1. Haffiner SM, Miettien H, Stem MP. The homeostasis model in the San Antonio Heart Study. Diabetes Care. 1997;20:1087-92.

2. Bogdanova K, Poczatkova H, Uherkova L, et al. Nonalcoholic fatty liver disease (NAFLD)-a novel common aspect of the metabolic syndrome. Biomed pap med Fac Univ Palacky Olomouc Czech Repub. 2006;150:101-4.

3. Meier U, Gressner AM. Endocrine regulation of energy metabolism: review of pathobiochemical and clinical chemical aspects of leptin, ghrelin, adiponectin, and resistin. Clin Chem. 2004;50:1511-25.

4. Umar RK, Rinella ME. Nonalcoholic fatty liver disease. Northwestern university, Chicago, Illinois. 2005;pp:94-8.

5. Pagano C, Sovado G, Esposito W, et al. Plasma adiponectin is decreased in nonalcoholic fatty liver disease. Clin. Endocrinol. Metab. 2005;90:3498-504. 
6. Bugianesi E, Pagotto U, Manini R, et al. Adiponectin in nonalcoholic fatty liver disease. The Journal of Clinical Endocrinology and Metabolism. 2005;90:3498-504.

7. Westerbacka J, Corner A, Tiikkainen M, et al. Women and men have similar amounts of liver and intra-abdominal fat, despite more subcutaneous fat in women: implications for sex differences in markers of cardiovascular risk. Diabetologia. 2004;47:1360-9.

8. Targher G, Bertolini L, Scala L, et al. Decreased plasma adiponectin concentrations are closely associated with nonalcoholic hepatic steatosis in obese individuals. Clin Endocrinol. 2004; Oxf 61:700-3.

9. Sargin H, Sargin M, Gozu H, et al. Nonalcoholic fatty liver disease and adiponectin. World J Gastroenterol. 2005;11:5874-7.

10. Kamada Y, Tamura S, Kiso S, et al. Enhanced carbon tetrachloride- induced liver fibrosis in mice lacking adiponectin. Gastroenterology. 2003;125:1796-807.

11. Yoon D, Lee SH, Park HS, et al. Hypoadiponectinemia and insulin resistance are associated with nonalcoholic fatty liver disease. J Korean Med Sci. 2005;20:421-6.
12. Yamamoto Y, Hirose H, Saito I, et al. Correlation of the adipocyte derived protein adiponectin with insulin resistance index serum high-density lipoprotein-cholesterol in the Japanese population. Clin Sci. 2002;103:137-42.

13. Bloomgarden ZT. Second world congress on insulin resistance syndrome: insulin resistance syndrome and nonalcoholic fatty liver disease. Diabetes Care. $2005 ; 28: 1518-23$.

14. Szczepaniak LS, Nurenberg P, Leonard D, et al. Magnetic resonance spectroscopy to measure hepatic triglyceride content: prevalence of hepatic steatosis in the general population. Am J Physiol Endocrinol Metab. 2005;288:E462-8

15. Marchesini G, Brizi M, Morselli-Labate AM, et al. Association of nonalcoholic fatty liver disease with insulin resistance. Am J Med. 1999;107:450-5.

16. Cusi. Long-term pioglitazone treatment for patients with nonalcoholic steatohepatitis and prediabetes or type 2 diabetes mellitus-A Randomized Trial. Ann Intern Med. 2016;165:305-15.

17. Olatunbosun S, Dagogo-Jack S. Insulin resistance. Emedecine. 2004;1-10.

\section{*Correspondence to:}

Hady Gad

Department of Internal Medicine

Middle East Medical Center

Kingdom of Bahrain

E-mail: hady_gad@yahoo.com 